\title{
Verschweigen wir es und stresst uns Corona doch?
}

\author{
Mirjam Tanner \\ Dr. med., Fachärztin für Psychiatrie und Psychotherapie, Leitungsausschuss ReMed
}

Das folgende Testimonial will zum Nachdenken und Diskutieren anregen - ganz ohne Fallgeschichte. Denn 2020 wurde das Unterstützungsnetzwerk ReMed für Ärztinnen und Ärzte kein einziges Mal zum Thema COVID-19 kontaktiert. Wir Beratenden sind darüber erstaunt und etwas beunruhigt. Bei der grossen Belastung der Ärzteschaft hatten wir eine Flut von Anfragen erwartet. Was steckt hinter diesem Schweigen?

Als die Schweiz Mitte März 2020 in den ersten CoronaLockdown ging, war die Situation für uns Ärztinnen und Ärzte mehr als herausfordernd. Entsprechend den Empfehlungen des Bundes fanden in unseren Arztpraxen nur noch Notfallkonsultationen statt - der Rest wurde vertagt, die Arbeit in Praxen und vielen Spitalabteilungen heruntergefahren. Das führte zu Verunsicherung, Ärger und nicht zuletzt zu wirtschaftlichen Einbussen. Lange Zeit gab es keine klare Richtlinie für die Umsetzung von Schutzkonzepten.

\section{Verunsicherung, hohes Ansteckungs- risiko und Isolation}

Das ReMed-Beratungsteam bekam mit, dass sich Ärzte in dieser schwierigen Lage in die Haare gerieten und bitter stritten. Es mangelte über Monate an medizinischen Schutzausrüstungen, Schutzkleidung und Desinfektionsmitteln. Angespannt erwarteten wir die erste grosse Welle an COVID-19-Patienten und bangten um die Kapazitäten auf unseren Intensivpflegeabteilungen und um die Gesundheit vulnerabler Angehöriger und Patienten.

Schliesslich, und das verfolgten wir mit Entsetzen und Mitgefühl, starben zu Beginn der Pandemie deutlich mehr Mitarbeitende des Gesundheitswesens, auch Ärztinnen und Ärzte, an COVID-19 als Angehörige anderer Berufsgruppen.

Die Pandemie zeigte uns Ärzten auf sehr direkte Weise, welchem erhöhtem Gesundheitsrisiko wir uns bei der Arbeit aussetzen. Inzwischen bestätigen auch neue Studien das erhöhte Risiko des Gesundheitspersonals, sich mit dem Virus anzustecken [1]. Zudem spürten auch wir die Folgen der Schutzmassnahmen und der damit einhergehenden Isolation an unseren Arbeitsplätzen. Es fehlten Möglichkeiten des Austauschs, sei es beim Mittagessen oder am Rande von Fortbildungen.

\section{Kaum stärkere Nachfrage nach Unterstützung}

Vor diesem Hintergrund erwarteten wir vom ReMedBeratungsteam ausgebrannte Kolleginnen und Kollegen aus der Intensivmedizin und anderen Disziplinen, die COVID-19 absorbierte. Entsprechend bereiteten wir uns auf eine erhöhte Nachfrage an Beratungen vor. Wir akquirierten mehr Personal und bauten das Angebot mit Coachinggruppen eigens für von COVID gezeichnete Kolleginnen und Kollegen aus. Wir nahmen an, dass sie uns jetzt noch dringender als sonst brauchen würden.

Doch im vergangenen Coronajahr führten wir nur 5\% mehr Beratungen durch als im Vorjahr und wurden nicht wie befürchtet von Anfragen überrannt. Corona wurde als Trigger von bestehenden Problemen genannt, nicht aber als spezifischer Grund einer Beratung. Das hat bei uns Fragen aufgeworfen und zum Nachdenken angeregt.

\section{Handeln als gesunde Antwort auf die Pandemie}

Was ist los mit uns Ärztinnen und Ärzten? Stresst Corona bloss alle anderen, aber uns nicht? Wie belastet müssen

\section{ReMed ist für Sie da}

Brauchen Sie oder jemand aus Ihrem Umfeld professionelle Hilfe? Wenden Sie sich an ReMed: Das Unterstützungsnetzwerk für Ärztinnen und Ärzte respektiert das Arztgeheimnis und berät Sie kompetent. Auch bei anderen beruflichen und persönlichen Krisen kann Ihnen ReMed Lösungswege aufzeigen. Dieses Angebot gilt auch für Personen aus dem Umfeld von Ärztinnen und Ärzten, 24 Stunden am Tag. Die ärztlichen Beratenden melden sich innerhalb von 72 Stunden: www.swiss-remed.ch, help[at] swissremed.ch, Tel. 0800073633. 


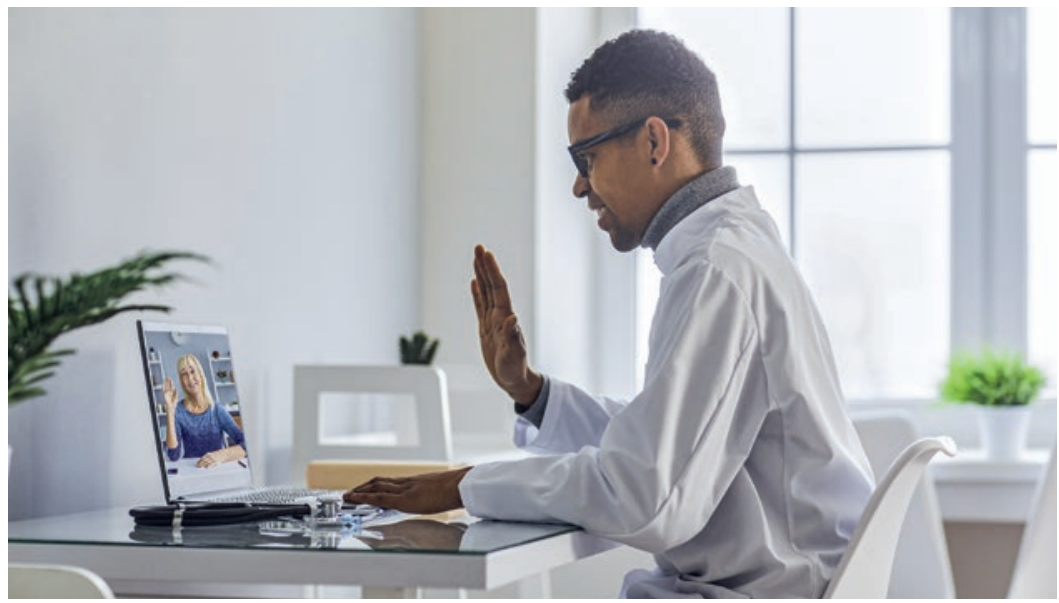

ReMed bietet u.a. eine moderierte Online-Coaching-Gruppe in deutscher Sprache zum lösungsfokussierten, interkollegialen Austausch über die aktuellen Herausforderungen an. Anmeldung per E-Mail an dr.s.werner[at]hin.ch

Ärzte sein, bis sie sich mitteilen, geschweige denn, sich Unterstützung holen? Ohne Zweifel, Intensivmediziner und auch andere Kolleginnen und Kollegen sind über sich hinausgewachsen, leisten einen immensen Einsatz bei jeder neuen Welle. Sie wurden im letzten Jahr zu Helden und oft ins Rampenlicht von Kliniken und Medien katapultiert - ob ihnen dies gefiel oder nicht.

So ist für sie die grosse Herausforderung durch Corona möglicherweise nicht der Moment des Burnouts. Vielleicht genauso wenig wie für alle anderen Kolleginnen und Kollegen, die in ihrem Alltag proaktiv, hilfreich und engagiert auf die Pandemie reagieren konnten.

\section{Und wenn kein aktives Handeln möglich ist?}

Wie aber ging und geht es allen anderen Ärztinnen und Ärzten? Jenen, die durch die Pandemie zu Passivität verknurrt und auf ein medizinisches Nebengleis gestellt wurden? Ich denke dabei an die niedergelassene Ärzteschaft genauso wie auch an Spitalärzte auf Abteilungen, die von Patienten plötzlich nicht mehr beansprucht wurden.

Neben dem wirtschaftlichen Druck: Was bedeutet es für eine Ärztin, einen Arzt, wenn sie beruflich nicht mehr «Vollgas» geben dürfen? Ist es nicht so, dass uns unser Commitment und grosser beruflicher Einsatz am Herzen liegen und unserem (beruflichen) Leben Wichtigkeit und Sinn verleihen? Was, wenn dies plötzlich nicht mehr zählt und bedeutungslos scheint?

Sabine Werner und

Pascale Kübler

ReMed

Postfach 300

CH-3000 Bern 15

Tel. 0313591200

info[at]swiss-remed.ch

\section{Schweigen bis 5 nach 12 - oder um 10 vor ReMed kontaktieren}

Corona hat uns immer noch im Griff. Die Auswirkungen des Pandemiestresses auf das Wohlbefinden und die mentale Gesundheit der Bevölkerung ist ein prominen- tes Thema in den Medien geworden. Was jedoch macht die Pandemie mit unserem eigenen Wohlbefinden und unserer eigenen mentalen Gesundheit als Ärztinnen und Ärzte? Wie viel Corona braucht es, bis es den Arzt selbst «juckt»? Oder macht uns die Pandemie zwar zu schaffen, aber wir erlauben es uns einfach nicht, für unsere Belastung, unseren Ärger, unsere Ängste und unseren Stress Worte zu finden und uns mitzuteilen? Dürfen wir uns selbst denn überhaupt eingestehen, Angst zu haben - beispielsweise uns mit dem Virus anzustecken oder finanzielle Verluste zu erleben [2, 3]? Können wir uns eingestehen, gekränkt zu sein, wenn wir plötzlich nicht mehr gleich gefragt sind, oder befürchten wir, als Narzissten wahrgenommen zu werden?

Vielleicht ist die fehlende Fallgeschichte dieses Testimonials einmal mehr Ausdruck eines Schweigens, das ReMed so gut kennt: Ärzte melden sich bei persönlichen Problemen bildlich gesprochen oft erst um "5 nach 12», wenn sie einschneidende Entscheidungen bereits einsam gefällt haben. Wir bei ReMed setzen uns dafür ein, dass keine Kollegin und kein Kollege sich schämen muss, auch dann nicht, wenn er oder sie sich rechtzeitig und bei "kleinem Jucken» an uns wendet. Das Unterstützungsnetzwerk ReMed kennt die Einsamkeit von Ärztinnen und Ärzten sehr gut und lässt keinen von ihnen im Stich.

\section{Bildnachweis}

Lacheev | Dreamstime.com

\section{Literatur}

1 Kramer et al. Subjective burden and perspectives of German healthcare workers during the COVID-19 pandemic. European Archives of Psychiatry and Clinical Neuroscience; 2/2021

2 Spiller TR et al. Development of health care workers' mental health during the SARS-CoV-2 pandemic in Switzerland: two cross-sectional studies. Psychological Medicine. 2020 Aug 13;1-4.

3 Weilenmann S et al. Health Care Workers' Mental Health During the First Weeks of the SARS-CoV-2 Pandemic in Switzerland. A Cross Sectional Study. Front. Psychiatry. 2021 (18 March).

\section{ReMed-Intervisionen für Erstberatende und Netzwerkmitglieder}

Neben den Unterstützungsangeboten für ratsuchende Ärztinnen und Ärzte führt ReMed auch regionale Intervisionen zum Erfahrungsaustausch für Kolleginnen und Kollegen durch, die Ärztinnen und Ärzte als Patienten betreuen. Diese ermöglichen Vernetzung und Bildung von Peer-Groups (jeweils 6-8 Teilnehmer, 2-3 Treffen pro Jahr), welche gemeinsam Fallfragen zu Mentoring, Coaching, Beratung, Therapie oder anderen Aspekten (juristisch, versicherungsrechtlich etc.) erarbeiten. Setzen Sie sich mit uns in Verbindung, nehmen Sie an einer Sitzung teil und lernen Sie unsere Arbeit kennen. Kontakt und Anmeldung: Dr. med. Sabine Werner, Mitglied Leitungsausschuss ReMed, dr.s.werner[at]hin.ch Mögliche nächste Daten 2021: 17.6.2021, 16.30-18 Uhr, Videokonferenz, sowie 16.9., 4.11., 11.11.2021, 14-18 Uhr, wenn möglich vor Ort im Hotel Plaza Zürich. 